\title{
Neuropragmatics: Brain and Communication
}

\author{
Bruno G. Bara and Maurizio Tirassa \\ Centro di Scienza Cognitiva, Università di Torino, Torino, Italy
}

Our field of research is cognitive pragmatics, that is, the theoretical and empirical study of the mental events involved in human communication. Communication is a form of social activity; more precisely, it is an agent's intentional and overt attempt to affect a partner's mental states (Airenti, Bara, \& Colombetti, 1993a, 1993b; Tirassa, 1997). While, historically, this area used to have no specific relationship with the neurosciences, our work is aimed at a convergence that seems to us capable of yielding valuable results.

Theories in pragmatics are typically analytical and developed on autonomous grounds; the neurosciences, and in particular the neuropsychology of mind/brain impairments, provide them with a natural empirical test bed. While, of course, the pathology of a cognitive function may always shed an interesting light on its physiology, in the case of communication this research strategy is made almost necessary by the intricacies of doing experimental pragmatics in the normal, healthy adult.

Different neuropsychological diseases will affect communicative performance in different ways, depending on what relevant cognitive subsystems are damaged and how. Competing theories of communication make different statements as to the nature of these subsystems and of their interconnections and therefore yield different predictions as to the patterns of their decay. It thus becomes possible to compare and to possibly falsify them. The same line of reasoning, transferred to normal development, also suggests to study the acquisition of communicative abilities in the child.

Neuroscience and pragmatics have quite distinct roles in this enterprise, the former being somewhat ancillary to the latter: given a powerful theory

This research is currently supported by the Italian Ministry of University and Scientific and Technological Research (MURST), Azione Integrata Italia/Spagna, 1999-2001.

Address correspondence and reprint requests to Bruno G. Bara, Centro di Scienza Cognitiva, Università di Torino, via Lagrange 3, 10123 Torino, Italy. E-mail: bara@psych.unito.it. 
of communication, all that the experimenters need is a reliable description of the particular neuropsychological syndrome they want to investigate, in order to be able to predict its consequences on the subjects' performance. The payoff for neuropsychology, however, would be a more precise, theoretically driven picture of what happens to the communicative skills of these patients and why. This would add in turn to our understanding of several diseases whose symptoms include a deficient communicative performance.

We have already brought about some research in this direction and at least another group has been using a similar perspective (Kasher, Batori, Soroker, Graves, \& Zaidel, 1999; Zaidel, Zaidel, Oxbury, \& Oxbury, 1995). Most of the work, however, remains to be done: we have only been able to test a few classes of subjects like head injured individuals (Bara, Tirassa, \& Zettin, 1997), Alzheimer's patients (Bara, Bucciarelli, \& Geminiani, 1999), and normal and neuropsychologically abnormal children (Bara, Bosco, \& Bucciarelli, 1999a, 1999b). Furthermore, our protocols have been more aimed at establishing, for each class of subjects, a basic trend of difficulty along which to order a few simple pragmatic phenomena than at exploring in detail how each type of neuropsychological damage actually affects communication.

While these considerations would suffice to guarantee the interest of a closer collaboration between pragmatics and neuropsychology, the most original and exciting results will likely come from a second and much more intimate relationship between them.

The cognitive sciences are currently undergoing an evolution that, in spite of the unpredictability of its outcome, is changing both the types of scientific questions that are asked and the very framework in which they are asked. The conception of the mind as the software of a digital computer is progressively losing ground in favor of its conception as the emerging property of the functioning of the brain. Correspondingly, as the classic computational philosophy and methodology decline, the connections of psychology with biology become stronger; in particular, with evolution theory, dynamic systems theory, and the neurosciences. Furthermore, to view the mind as the evolved control system that governs an organism's interactions with the world, instead of a rational mechanism devoted to abstract symbol manipulation, brings previously neglected issues (e.g., developmental, social, and clinical ones) into focus. Areas like neuropsychology and pragmatics may play a pivotal role in this evolution, provided that they are prepared and equipped to do so.

Theories of pragmatics are typically cast in terms of the sequences of mental states (like beliefs and intentions) that two agents entertain as they plan and understand the communicative acts that make up their dialogue. Arguably, this is the only possible paradigm for the very definition of human communication. In a biologically oriented framework, mental states are viewed as features of the brain. When it comes to the role and the future developments of neuroscience, however, this is no trivial doctrine, since it 
implies the rejection of any reductionist stance and the acknowledgment of a causal role of consciousness in brain functioning (Tirassa, 1999a).

The import of a nondualist and noneliminativist position is not merely philosophical: both the formal structure and the empirical consequences of a theory cast in terms of mental states may differ profoundly from those of a classic cognitive one.

While, for example, most researchers would not subscribe to a Fodorian view of the mind/brain in terms of general-purpose central systems plus rigidly encapsulated input modules any longer, it is far from clear what alternative architectures should be considered. Compelling evidence from neuroscience, evolutionary psychology, and developmental psychology is in favor of a domain-specific architecture; this, however, cannot be conceived of as a network of Fodorian modules, because such modules can only exist in the service of the Language of Thought, that is, of precisely the domain-general central systems whose existence is questioned.

A hint to a possible solution comes from the view of communication as competence, that is, as a distinct, innate cognitive faculty that governs a specific domain of agent/world interaction (Tirassa, 1999b). Communicative competence is not a Fodorian module, at least because it is intentional (that is, representational) and because it is not encapsulated (that is, it does not function independently of the rest of what is going on in the agent's mind).

This approach makes it possible to cope with otherwise unsolved problems like the nature of communicative meanings and actions (Bara \& Tirassa, 1999) and the innate bases of communication in the human species (Bosco \& Tirassa, 1998), which may help in turn to settle more general problems like the implausibility of tabula rasa perspectives on mind/brain development. It also poses, however, new questions: for example, how should the relationships between communication and other cognitive competencies like planning be conceived of? What is an inference in understanding, if it is not the application of a general rule of deduction? What is the functional role of brain areas specialization when a view of communication as the flow of domain-specific mental states is substituted for a boxes-and-arrows paradigm?

The answer to these and similar questions can only be looked for in a truly interdisciplinary framework, with the neurosciences playing a crucial role in the endeavor.

This does not necessarily mean that sophisticated machinery ought to be used. Brain-imaging technology, to mention one, can hardly replace old fashioned bedside methodologies. If communication is a complex activity and one that is governed by a representational competence rather than an encapsulated module, it will probably involve a complex network of brain locations: no single, fixed cell for giving socially appropriate responses in conversation is likely to be found.

For the same reason, to investigate communication requires that unconventional types of experimental tasks be devised: the measurement of reac- 
tion times, for example, is substantially irrelevant, and even the judgment of what counts as a correct answer or a good performance is an intricate matter. In our work on neuropragmatics, we tested the comprehension of videotaped scenes reproducing simple everyday situations, an approach that is certainly more reminiscent of Frederick Bartlett than of what is generally filed under "frontiers of neuroscience."

A final remark is that a more ecological framework for the study of the mind/brain in terms of situated (that is, interaction-centered and contextaware) macroprocesses ought to substitute for the current one, that typically builds on artificially isolated microprocesses instead. We think that the time has come to abandon the parceling approaches that were typical of information-processing psychology and the consequent construction of theoretical and empirical microworlds. The neurosciences will provide inestimable results, once the real problems that the real human beings have to cope with are taken into account.

The picture we envisage will be clear by now. Communication is interesting both in itself and as a crucial part of what human beings do in the real world; furthermore, the questions that its study poses require that stances be taken with respect to several fundamental problems of the cognitive sciences. Neuroscience may give a crucial contribution to the shaping and the framing of these questions and problems. The payoffs of a better integration in the study of the mind/brain will be the knowledge acquired and, even more important, a richer perspective on what human beings are.

\section{REFERENCES}

Airenti, G., Bara, B. G., \& Colombetti, M. 1993a. Conversation and behavior games in the pragmatics of dialogue. Cognitive Science, 17, 197-256.

Airenti, G., Bara, B. G., \& Colombetti, M. 1993b. Failures, exploitations and deceits in communication. Journal of Pragmatics, 20, 303-326.

Bara, B. G., Bosco, F. M., \& Bucciarelli, M. 1999a. Simple and complex speech acts: What makes the difference within a developmental perspective. In M. Hahn \& S. C. Stoness (Eds.), Proceedings of the 21st annual conference of the cognitive science society (Vancouver, BC). Mahwah, NJ: Erlbaum.

Bara, B. G., Bosco, F. M., \& Bucciarelli, M. 1999b. Developmental pragmatics in normal and abnormal children. Brain and Language, 68, 507-528.

Bara, B. G., Bucciarelli, M., \& Geminiani G. (in press). Development and decay of extralinguistic communication. Brain and Cognition.

Bara, B. G., \& Tirassa, M. 1999. A mentalist framework for linguistic and extralinguistic communication. In Proceedings of the 3rd European Conference on Cognitive Science (ECCS '99) Siena, Italy.

Bara, B. G., Tirassa, M., \& Zettin, M. 1997. Neuropragmatics: Neuropsychological constraints on formal theories of dialogue. Brain and Language, 59, 7-49.

Bosco, F. M., \& Tirassa, M. 1998. Sharedness as an innate basis for communication in the infant. In M. A. Gernsbacher \& S. J. Derry (Eds.), Proceedings of the 20th annual conference of the cognitive science society (Madison, WI). Mahwah, NJ: Erlbaum. 
Kasher, A., Batori, G., Soroker, N., Graves, D., \& Zaidel, E. 1999. Effects of right and left hemisphere damage on understanding conversational implicatures. Brain and Language, 68, 566-590.

Tirassa, M. 1997. Mental states in communication. In Proceedings of the 2nd European Conference on Cognitive Science (ECCS '97) Manchester, UK.

Tirassa, M. 1999a. Taking the trivial doctrine seriously: Functionalism, eliminativism, and materialism. Behavioral and Brain Sciences, in press.

Tirassa, M. 1999b. Communicative competence and the architecture of the mind/brain. Brain and Language, 68, 419-441.

Zaidel, D. W., Zaidel, E., Oxbury, S. M., \& Oxbury, J. M. 1995. The interpretation of sentence ambiguity in patients with unilateral focal brain surgery. Brain and Language, 51, 458468. 